\title{
ERRATUM
}

\section{Celastrol, an active constituent of the TCM plant Tripterygium wilfordii Hook.f., inhibits prostate cancer bone metastasis}

\author{
K Kuchta, Y Xiang, S Huang, Y Tang, X Peng, X Wang, Y Zhu, J Li, J Xu, Z Lin and T Pan
}

Prostate Cancer and Prostatic Diseases (2017) 20, 250; doi:10.1038/pcan.2017.11; published online 28 March 2017

Correction to: Prostate Cancer and Prostatic Diseases (2017) advance online publication 14 February 2017; doi:10.1038/pcan. 2016.61

The corresponding author and author contributions were not accurately represented in the published version of this paper. $\mathrm{T}$ Pan should have been listed as the corresponding author. $K$ Kuchta and $Y$ Xiang should have been denoted as sharing equal contribution. The correct corresponding author information is listed below. The publisher regrets the errors.

Corresponding author: Prof. T Pan, Department of Orthopaedic Surgery, the Sixth Affiliated Hospital of Sun Yat-sen University, 26 Yuancun Er Heng Road, Guangzhou, Guangdong 510655, China, Phone: +86-20-38254211, Fax: +86-20-38254221.

E-mail: pantao@126.com 